\title{
Über die Wirkung von Ammoniak und Ammoniakderivaten auf die Oxydationsprozesse in Zellen.
}

\author{
Von \\ Eduard Grafe.
}

(Der Redaktion zugegangen am 31. Mai 1912.)

(Aus der medizinischen Klinik in Heidelberg.)

Als Fortsetzung der Untersuchungen über Oxydationsbeeinflussungen soll im folgenden über die Wirkung des Ammoniaks, der einfachen Amine und der komplizierteren Amine (Alkaloide) berichtet werden. Die Versuchsanordnung war dieselbe, wie sie in früheren Arbeiten ausführlich beschrieben worden ist. ${ }^{1}$ ) Herr Dr. Warburg hat mich zur Ausführung dieser Untersuchung angeregt und bei ihr angeleitet.

Ein bemerkenswerter Unterschied im Verhalten der Zellen gegen basische Substanzen besteht darin, da $B$ beim Vermischen und Waschen der Zellsuspensionen mit basischen Lösungen Gleichgewichte nicht erreicht werden. Mischt man z. B. $2 \mathrm{ccm}$ Zellsuspension (in physiologischer Kochsalzlösung) mit $15 \mathrm{ccm}$ 1/200-n-Ammoniaklösung (gleichfalls in physiologischer Kochsalzlösung), zentrifugiert und bestimmt die $\mathrm{OH}-$ Ionenkonzentration in der überstehenden Flüssigkeit, so findet man sie bedeutend geringer, als einer Verdünnung von $15: 17 \mathrm{~cm}$ entsprechen würde. Mit Hilfe von Indikatoren kann man sich leicht hiervon überzeugen. Die Alkalescenzabnahme, die sofort und auch bei $0^{0}$ eintritt, findet jedesmal beim Vermischen mit neuen Quantitäten Basenlösung wieder statt, wobei die Ursache zunächst u.a. eine Speicherung von Ammoniak in der Zelle ist; später nimmt nicht mehr die $\mathrm{NH}_{3}$-Menge, sondern nur noch die $\mathrm{OH}$-Ionenkonzentration in der überstehenden Flüssigkeit ab, wie kolori-

1) Warburg, Zeitschrift f. physiol. Chemie, Bd. 76, S. 331 (1912). Warburg u. Wiesel, Pflügers Archiv, Bd. 144, S. 465. Ferner eine kurze Mitteilung über Wirkung basischer Substanzen. O. Warburg, Verhandlungen des Deutschen Kongresses für innere Medizin 1911, S. 553.

Hoppe-Seyler's Zeitschrift f. physiol. Chemie. LXXIX. 
metrische Ammoniakbestimmungen nach Neßler lehren. Wäscht man fortgesetzt mit selbst äußerst verdünnten Ammoniaklösungen, so gehen die Zellen unter Farbstoffaustritt zugrunde. Ähnlich wie Ammoniak verhalten sich die einfacheren und zum Teil auch die komplizierteren Amine.

Unter diesen Verhältnissen hängt es völlig von der Menge der Basenlösung bezw. von der Zahl der Waschungen ab, mit welcher Konzentration eine Wirkung erzielt wird, je nach der Anordnung läßt sich mit ${ }^{1 / 100}$ oder $1 / 1000-n-A m m o n i a k$ das Gleiche erreichen und es entsteht die Frage, ob zahlenmäßigen Angaben überhaupt ein Wert zukommt. Diese Frage muß bejaht werden, weil bei einigermaßen ähnlichem Vorgehen die Regelmäßigkeit der Resultate völlig befriedigend ist. Versuche, die im Laufe der letzten 1/2 Jahre zu verschiedenen Jahreszeiten, mit verschiedenstem Zellmaterial angestellt wurden, verliefen ausnahmslos gleich, sodaß die Versuchsbedingungen als ebensogut reproduzierbar bezeichnet werden müssen, wie diejenigen, bei denen es sich um Gleichgewichtskonzentrationen handelt.

Die Experimente können entweder so angeordnet werden, daß die Konzentrationen der Basenlösungen oder bei gleichen Konzentrationen die Mengen der Basenlösungen variiert werden. Im folgenden wurde meist nach dem zweiten Modus verfahren, und je nachdem mit einer Basenlösung bestimmter Konzentration einmal, zweimal oder mehrmals gewaschen wurde, konnte die ganze Skala der Basenwirkung beobachtet werden, d. h. bei kleinster Menge Steigerung der Oxydationsprozesse, bei größerer Menge reversible Hemmung, bei noch größerer Menge irreversible Hemmung und schließlich Hämolyse. Diese Stufenfolge ist charakteristisch für die einfachen Amine bis hinauf zum Coniin und Nicotin, während bei den komplizierteren Aminen mit hohem Molekulargewicht die eine oder andere Erscheinung ausfällt, wofür teilweise die Löslichkeitsverhältnisse verantwortlich zu machen sind.

Worin besteht nun das Wesen der Aminwirkung? Im Ammoniak sind im chemischen Sinn reaktionsfähig die Wasserstoffatome der Amidogruppe und man könnte wohl erwarten, daß ein Zusammenhang dieser reaktionsfähigen Atome mit der 
Wirkung auf die chemischen Prozesse in der Zelle besteht, daß also beispielsweise tertiäre Amine, in denen diese Wasserstoffatome substituiert sind, schwächer oder anders als Ammoniak reagieren; daß Allylamin vermöge seiner Doppelbindung Unterschiede zeigt gegenüber der gesättigten Verbindung, dem Propylamin usw. Das ist nicht der Fall.

Für eine große oder vielleicht die größte Zahl der Kohlenstoffverbindungen wurde eine Abhängigkeit der Wirkungsstärke von gewissen physikalischen Eigenschaften, die im Zusammenhang mit ihrem Teilungsverhältnis zwischen Öl und Wasser stehen, festgestellt. Diese Beziehung besteht nicht für die Amine, Substanzen mit so verschiedenem Teilungsverhältnis, wie Ammoniak und Piperidin einerseits, Amylamin und Coniin anderseits, wirken fast gleich.

Anordnung: $2 \mathrm{ccm}$ Zellsuspension $+10 \mathrm{ccm}$ Basenlösung.

Tabelle I.

\begin{tabular}{|c|c|c|c|}
\hline Substanz & & $\begin{array}{l}\text { Hemmend } \\
\text { g Mol. } \\
\text { pro Liter }\end{array}$ & $\begin{array}{c}\text { Dissoziation in } \% \\
\text { in } 1 / 288-n-L o ̈ u n g ~ n a c h \\
\text { Bredig }\end{array}$ \\
\hline Ammoniak . . & . • . & $1 / 50$ & 7,5 \\
\hline Monomethylamin & . . . & $1 / 100$ & 29 \\
\hline Dimethylamin . . & . . . & $1 / 100$ & 35 \\
\hline Trimethylamin . & . . . & $1 / 50$ & 13 \\
\hline Triäthylamin . . & . . . & $1 / 100$ & 33 \\
\hline Propylamin . . . & . . . & $1 / 100$ & 29 \\
\hline Allylamin . . . & . . . . & $1 / 76$ & 11,4 \\
\hline Amylamin . . . & . . . . & $1 / 100$ & 29,4 \\
\hline Benzylamin . . . & . . & $1 / 50$ & 7,6 \\
\hline Piperidin . . . . & . . . & $1 / 100$ & 46 \\
\hline Coniin . . . . & . . . & $1 / 100$ & 43 \\
\hline Nicotin. . . & . . & $1 / 35$ & $>7,5$ \\
\hline
\end{tabular}

Einzig und allein ausschlaggebend ist offenbar die Konzentration der OH-Ionen im wässerigen Teil 
der Zelle. Ein Blick auf vorstehende Tabelle lehrt erstens, daß die Wirkungsstärke der verschiedenartigsten Amine ihrer Größenordnung nach die gleiche ist, zwischen Ammoniak, Trimethylamin, Nicotin, Allylamin sind, im Vergleich zu den enormen Unterschieden, die bei neutralen Substanzen gefunden wurden, nur ganz geringfügige Differenzen. Vergleicht man nun die (nebenstehenden) Dissoziationskonstanten, so ist eine Beziehung zu den Wirkungsstärken ganz unverkennbar, während die Tatsache, daß die Größenordnung der Wirkungsstärken die gleiche ist, ihre befriedigende Erklärung darin findet, daß auch die Größenordnung der Dissoziation dieser Substanzen gleich ist.

Gleichzeitig folgt, daß chemische Reaktionen, die nach den Formeln etwa denkbar wären, in der Zelle entweder nicht vor sich gehen oder jedenfalls für die Wirkung belanglos sind.

In der zweiten Tabelle ist die Anordnung so gewählt, daß $2 \mathrm{ccm}$ Zellsuspension etwa dreimal mit $16 \mathrm{ccm}$ Basenlösung gewaschen wurden. In Übereinstimmung mit den obigen Erörterungen sind dann die absoluten wirksamen Konzentrationen erheblich niedriger, während dieselben relativen Verhältnisse wie in Tabelle I bestehen.

Auf eine Theorie dieser Wirkungen einzugehen, ist sicherlich verfrüht, doch sei im Zusammenhang mit dem merkwürdigen gegensätzlichen Verhalten verschiedener $\mathrm{OH}-\mathrm{Ionenkonzentrationen} \mathrm{-} \mathrm{Steigerung} \mathrm{der} \mathrm{Oxydations-}$ prozesse bei kleinen, reversible Hemmung bei größeren Konzentrationen - daran erinnert, daß die Wirkung der OH-Ionen auf kolloidchemische Phänomene vielfach durch ein Maximum geht. ${ }^{1}$ )

Die komplizierteren Amine (Alkaloide) wirken deutlich stärker, als ihren Dissoziationskonstanten entspricht, wie folgende Beispiele zeigen (Tabelle III).

1) Freundlich, Über Adsorption in Lösungen. Zeitschrift für physikalische Chemie, Bd. 57, S. 461 (1906). Bredig, ibid., Bd. 31, S. 258 (1899). 
Tabelle II.

\begin{tabular}{|c|c|c|}
\hline Substanz & $\begin{array}{l}\text { g Mol. } \\
\text { pro } \\
\text { Liter }\end{array}$ & $\begin{array}{l}\text { Oxydations- } \\
\text { hemmung } \\
\text { in } \%\end{array}$ \\
\hline Ammoniak . & $1 / 100$ & 79 (Vers. 5) \\
\hline Methylamin . & $1 / 200$ & $84(>8)$ \\
\hline Dimethylamin & $1 / 200$ & $82(, 11)$ \\
\hline Propylamin & $1 / 200$ & 76( \\
\hline Allylamin . & $1 / 200$ & $73(, 12)$ \\
\hline Amylamin . & $1 / 200$ & $77(, 10)$ \\
\hline Nicotin . & $1 / 50$ & 62( \\
\hline Coniin . & $1 / 200$ & 57 \\
\hline
\end{tabular}

Tabelle III.

\begin{tabular}{|c|c|c|}
\hline Substanz & $\begin{array}{l}\text { g Mol. } \\
\text { pro } \\
\text { Liter }\end{array}$ & $\begin{array}{l}\text { Oxydations- } \\
\text { hemmung } \\
\text { in } \% / 0\end{array}$ \\
\hline Brucin . . & $\mathrm{n} / 200$ & 64 (Vers. 14) \\
\hline Chinin . . & $1 / 1000$ & $76(>15)$ \\
\hline Codein . . . & $n / 400$ & $36(, 16)$ \\
\hline Cocain . . & $n / 400$ & $43(, 17)$ \\
\hline Atropin . & $\mathrm{n} / 400$ & $90(, 18)$ \\
\hline
\end{tabular}

Anhangsweise sind noch Versuche mit einigen pharmakologisch wichtigen Substanzen: Coffein, Antipyrin, Veronal u. a. beigefügt. Bemerkenswert ist vielleicht, daß Veronal, das in 1/s0-n-Lösung deutlich hemmt, in neutraler Lösung fast wirkungslos ist; auf Grund der Overtonschen Plasmahauttheorie ohne weiteres verständlich, denn Diäthylbarbitursäure ist lipoidlöslich, das Natronsalz, wie die meisten Salze, lipoidunlöslich.

\section{Methodik.}

Die Versuche sind wieder mit roten Blutzellen anämischer Gänse ausgeführt und in fast allen Einzelheiten die Vorschriften befolgt, die in dieser Zeitschrift, Bd. 76, S. 331 gegeben wurden.

Die Druckverminderung ohne Atmung wurde diesmal nicht mit einer Cyanidkontrolle bestimmt, sondern die Hemmung war durch Ammoniak oder andere Basen bewirkt. Um sicher zu sein, daß das Serum beim Waschen in Kochsalzlösung hinreichend entfernt war, wurde so lange gewaschen, bis die überstehende Flüssigkeit gegen Neutralrot sauer reagierte. Stets wurden $2 \mathrm{ccm}$ konzentrierte Zellsuspension verwendet. Unter «Waschen» ist im folgenden Auffüllen von $2 \mathrm{ccm}$ Zellsuspension auf $18-20 \mathrm{ccm}$ verstanden, und wieder Abhebern der überstehenden Flüssigkeit auf ca. $2 \mathrm{ccm}$.

Versuch 1-4 zeigt, daß physiologische Kochsalzlösung die Atmung in längeren Zeiten vermindert. 
Solche längere Versuchszeiten kommen in Betracht, wenn geprüft werden soll, ob eine Hemmung reversibel ist. Für diese Experimente wurde deshalb eine Art Lockesche Lösung verwendet, in der die Atmung hinreichend konstant bleibt.

Die Temperatur des Thermostaten, in dem die Atmungsversuche vorgenommen wurden, betrug etwa $29^{\circ}$.

:. Die Basenlösungen wurden entweder aus den Salzen durch Zufügung kohlensäurefreier Natronlauge hergestellt; ${ }^{1}$ ) stand nur die freie Base zur Verfügung, so wurde sie mit überschüssiger Salzsäure angesäuert, einige Minuten geschüttelt und die Salzsäure wieder genau mit kohlensäurefreier Natronlauge neutralisiert. Mit konzentrierter Natriumchloridlösung wurde dann der physiologische osmotische Druck bezüglich Natriumchlorid hergestellt.

\section{Versuche.}

\section{A. Untersuchung von Salzlösungen.}

1. Physiologische NaCl-Lösung.

Versuch 1. \& Blutsuspensionen von je $2 \mathrm{ccm}$ wurden mit $0,9 \%$ NaCl-Lösung auf $10 \mathrm{ccm}$ aufgefüllt und für eine bestimmte Zeit in den Thermostaten von $29^{\circ}$ gebracht.

4 Blutsuspensionen von je $2 \mathrm{ccm}$ wurden ebenso behandelt, dann mehrmals mit $0,9 \% \mathrm{NaCl}$-Lösung gewaschen und für die gleiche Zeit nochmals in den Thermostaten von $29^{\circ}$ gebracht:

I mit $0,9 \% \mathrm{NaCl}$ aufgefüllt und für $2 \mathrm{Std}$. $30 \mathrm{Min}$. in den Thermostaten.

Ia wie I behandelt, dann 3 mal mit ca. $20 \mathrm{ccm}$ NaCl-Lösung gewaschen und für $2 \mathrm{Std}$. $30 \mathrm{Min}$ in den Thermostaten.

II mit $0,9 \% \mathrm{NaCl}$ aufgefüllt, für $1 \mathrm{Std}$. $45 \mathrm{Min}$. in den Thermostaten.

II a wie II, dann 2 mal gewaschen und für 1 Std. 45 Min. in den Thermostaten.

III mit 0,9\% NaCl aufgefültt; Dauer 1 Std. 30 Min.

III a wie III, dann 2 mal gewaschen und für $1 \mathrm{Std}$. $30 \mathrm{Min}$. in den Thermostaten.

IV mit $0,9 \% \mathrm{NaCl}$ aufgefüllt; Dauer $2 \mathrm{Std}$.

IV a wie III, dann 2 mal gewaschen und für 2 Std. in den Thermostaten.

1) Sörensen, Biochemische Zeitschrift, Bd. 21 (1909)

$$
\begin{aligned}
& 250 \text { g } \mathrm{NaOH} \\
& 300 \text {, Wasser }
\end{aligned}
$$

nach einigen Tagen das überstehende Wasser abpipettieren. 


\begin{tabular}{ll|c|c|c|c}
\hline & v. & t. & p. & $\begin{array}{c}\text { Sauerstoff- } \\
\text { verbrauch } \\
\text { in ccm }\end{array}$ \\
\hline I erste 2 Std. 30 Min. & 37 & 16 & -127 & 0,44 \\
I a zweite 2 $>30$, & 37 & 16 & -75 & 0,26 \\
\hline II erste 1 Std. 45 Min. & 37 & 16 & -142 & 0,50 \\
II a zweite 1 > 45 & 37 & 16 & -84 & 0,29 \\
\hline III erste 1 Std. 30 Min. & 37 & 16 & -58 & 0,20 \\
III a zweite 1 $>30$, & 37 & 16 & -38 & 0,13 \\
\hline IV erste 2 Std. & 37 & 16 & -92 & 0,32 \\
IV a zweite 2 > & 37 & 16 & -53 & 0,18
\end{tabular}

Resultat: Bei mehrstündigem Aufenthalt in $0,9 \% \mathrm{NaCl}$ sinkt die Oxydationsgröße erheblich ab.

\section{Physiologische NaCl-Lösung.}

Versuch 2 soll die Frage beantworten, ob mehrmaliges Waschen

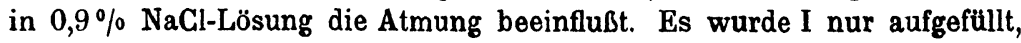
II 2 mal mit NaCl-Lösung gewaschen, III 4 mal mit NaCl-Lösung gewaschen, I, II und III kamen dann für $1 \mathrm{Std}$. $30 \mathrm{Min}$. in den Thermostaten.

\begin{tabular}{c|c|c|c|c}
\hline & v. & t. & p. & $\begin{array}{c}\text { Sauerstoff- } \\
\text { verbrauch } \\
\text { in ccm }\end{array}$ \\
\hline I Nur aufgefüllt mit $\mathrm{NaCl}$ & 37 & 16 & -89 & 0,31 \\
II 2 mal gewaschen mit NaCl & 37 & 16 & -82 & 0,29 \\
III 4 , > & 37 & 16 & -85 & 0,30
\end{tabular}

Resultat: Mehrmaliges Waschen in $0,9 \%$ Kochsalzlösung beeinflußt die Atmung nicht bemerkenswert.

3. Lockesche Lösung ohne $\mathrm{NaHCO}_{3}$.

Versuch 3. Lösung pro Liter:

$$
\begin{array}{ll}
8,0 \mathrm{~g} & \mathrm{NaCl}, \\
0,3 & \rightarrow \mathrm{CaCl}_{2}, \\
0,3 & =\mathrm{KCl}, \\
5,0 & \rightarrow \text { Traubenzucker. }
\end{array}
$$

Wir bezeichnen diese Lösung im folgenden als modifizierte Lock e-L.

An ordnung: I mit NaCl aufgefüllt, II mit modifizierter Locke-L. aufgefüllt, beide $2 \mathrm{Std}$. $5 \mathrm{Min}$. in den Thermostaten.

Ia wie I, dann 3 mal mit $\mathrm{NaCl}$ gewaschen.

II a wie II, dann 3 mal mit modifizierter Locke gewachen; I a und II a nach dem Waschen nochmals für 2 Std. 5 Min. in den Thermostaten. 


\begin{tabular}{c|c|c|c|c}
\hline \hline & v. & t. & p. & $\begin{array}{c}\text { Sauerstoff- } \\
\text { verbrauch } \\
\text { in ccm }\end{array}$ \\
\hline $\mathrm{NaCl}$ erste Periode ...... & 37 & 16 & -143 & 0,50 \\
$\mathrm{NaCl}$ zweite Periode ..... & 37 & 16 & -78 & 0,27 \\
\hline mod. Locke erste Periode... & 37 & 16 & -145 & 0,51 \\
...+ & 37 & 16 & -107 & 0,37
\end{tabular}

Resultat: Die mit Lockescher L. und $\mathrm{NaCl}$ aufgefüllten Suspensionen haben in der ersten Periode von 2 Std. 5 Min. gleich stark geatmet. In der zweiten Periode ist ein deutlicher Abfall zu konstatieren, der für mod. Locke L. geringer als für $\mathrm{NaCl}$ ist.

4. Lockesche Lösung mit $\mathrm{NaHCO}_{3}$.

Versuch 4. Lösung pro Liter:

$$
\begin{aligned}
7,2 & \text { g } \mathrm{NaCl}, \\
1,0 & >\mathrm{NaHCO}_{3}, \\
0,3 & >\mathrm{CaCl}_{2}, \\
0,3 & >\mathrm{KCl}, \\
5,0 & >\text { Traubenzucker. }
\end{aligned}
$$

\begin{tabular}{|c|c|c|c|c|}
\hline & v. & $\mathrm{t}$. & p. & $\begin{array}{l}\text { Sauerstoff- } \\
\text { verbrauch } \\
\text { in } \mathrm{ccm}\end{array}$ \\
\hline $\mathrm{NaCl}$ erste Periode . . . . & 37 & 16 & -74 & 0,26 \\
\hline $\mathrm{NaCl}$ zweite Periode . . & 37 & 16 & -54 & 0,19 \\
\hline Locke erste Periode $\ldots \ldots$ & 37 & 16 & -70 & 0,24 \\
\hline Locke zweite Periode . . . . & 37 & 16 & -71 & 0,25 \\
\hline
\end{tabular}

Anordnung: wie in Versuch 3, Dauer einer Periode 90 Min.

Resultat: Eine Schädigung der Atmung durch Lockesche Lösung mit $\mathrm{NaHCO}_{3}$ findet nicht statt.

\section{B. Untersuchung von Ammoniak und Ammoniakderivaten.}

\section{Ammoniak.}

Versuch 5: Ammoniak.

Bereitung der Lösung: $\mathrm{Zu} 0,6 \mathrm{~g} \mathrm{~N} \mathrm{NH}_{4} \mathrm{Cl}$ (berechnet $0,54 \mathrm{~g}$ ) werden $100 \mathrm{ccm} 1 / 10-\mathrm{n}-\mathrm{NaOH}$ zugesetzt. Es fehlen dann bis zum physiologischen 
Gehalt der Lösung noch $0,3 \mathrm{~g} \mathrm{NaCl}$, die in $1,5 \mathrm{ccm}$ einer $20 \% \mathrm{NaCl}$ Lösung zugegeben werden. Wir erhalten so $1 / 10-n-\mathrm{NH}_{3}$ in physiologischer Kochsalzlösung. Diese Lösung, auf das 10 fache mit $\mathrm{NaCl}(0,9 \%)$ verdünnt, hat eine Konzentration von 1/100-n.

Anordnung: Je $2 \mathrm{ccm}$ einer konzentrierten Zellsuspension werden: I 3 mal mit $1 / 100-n-N_{3}$ gewaschen, dann auf $10 \mathrm{ccm}$ aufgefüllt. II mit $1 / 100-\mathrm{n}-\mathrm{NH}_{3}$ auf $10 \mathrm{ccm}$ aufgefüllt.

III mit $0,9 \% \mathrm{NaCl}$ auf $10 \mathrm{ccm}$ aufgefüllt.

\begin{tabular}{c|c|c|c|c}
\hline \hline & v. & t. & p. & $\begin{array}{c}\text { Sauerstoff- } \\
\text { verbrauch } \\
\text { in ccm }\end{array}$ \\
\hline I 3 mal gewaschen & 37 & 16 & -15 & 0,052 \\
II aufgefüllt & 37 & 16 & -116 & 0,41 \\
III NaCl & 37 & 16 & -74 & 0,26
\end{tabular}

Dauer des Versuchs 3 Stunden.

Resultat: $1 / 100-n$ hemmt um $79 \%$ gegen $\mathrm{NaCl}$.

$$
\text { 6. Coniin }(M=127) \text {. }
$$

Versuch 6. Coniin.

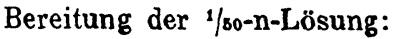

$$
\begin{aligned}
0,3 \mathrm{ccm} & (=0,25 \mathrm{~g}) \text { Coniin, } \\
40,0 & \searrow 1 / 10-\mathrm{n}-\mathrm{HCl}, \\
3,4 & >20 \% \mathrm{NaCl}-L o ̈ s u n g=0,68 \mathrm{~g} \mathrm{NaCl}, \\
43,2 & \left.>1 / 10-\mathrm{n}-\mathrm{NaOH},{ }^{1}\right) \\
13,0 & >\mathrm{H}_{\mathbf{2}} \mathrm{O} .
\end{aligned}
$$

$100 \mathrm{ccm} n / 50-n$ auf $400 \mathrm{ccm}$ mit NaCl verdünnt, geben n/200-Coniin.

Anordnung:

I 3 mal gewaschen und frisch aufgefüllt; wird hämolytisch.

II 1 mal gewaschen und frisch aufgefüllt.

III Nur aufgefüllt mit n/200-Coniin.

IV NaCl-Kontrolle.

\begin{tabular}{c|c|c|c|c}
\hline & v. & t. & p. & $\begin{array}{c}\text { Sauerstoff- } \\
\text { verbrauch } \\
\text { in ccm }\end{array}$ \\
\hline II 1 mal gewaschen und & 37 & 17 & -39 & 0,14 \\
frisch aufgefüllt & 37 & 17 & -147 & 0,51 \\
III nur aufgefüllt & 37 & 17 & -92 & 0,32 \\
IV NaCl &
\end{tabular}

Dauer des Versuchs 3 Std. 15 Min.

1) $43,2 \mathrm{ccm}$ unserer $\mathrm{NaOH}$ neutralisierten $40,0 \mathrm{ccm} \mathrm{n} / 10-\mathrm{HCl}$. 
Resultat: n/200-Coniin hemmt die Atmung bei einmaligem Waschen und frischem Auffüllen um $\mathbf{5 7} \%$ gegen $\mathrm{NaCl}$.

7. Propylamin.

Versuch 7: Propylamin.

Bereitung der Lösung:

1. Herstellung von $1 / 50-n$-Propylamin wie im vorigen Versuch.

2. Verdünnung auf $400 \mathrm{ccm}$ ergibt $\mathrm{n} / \mathbf{2 0 0}$-Propylamin.

Anordnung:

I 3 mal gewaschen.

II 1 mal gewaschen und frisch aufgefüllt.

III nur aufgefüllt mit $\mathrm{n} / \mathbf{2 0 0}$-Propylamin.

IV NaGl-Kontrolle.

\begin{tabular}{c|c|c|c|c}
\hline & v. & t. & p. & $\begin{array}{c}\text { Sauerstoff- } \\
\text { verbrauch } \\
\text { in ccm }\end{array}$ \\
\hline I 3 mal gewaschen & 37 & 17 & -21 & 0,073 \\
III aufgefüllt & 37 & 17 & -124 & 0,43 \\
IV NaCl & 37 & 17 & -90 & 0,31
\end{tabular}

Dauer des Versuchs: 2 Std. 45 Min.

Resultat: $\mathbf{n} / \mathbf{2 0 0}$-Propylamin hemmt bei 3 maligem Waschen die Atmung um $76 \%$.

8. Methylamin.

Verwandt wird $\mathrm{n} / \mathrm{so0}$.

Anordnung:

I 3 mal gewaschen.

II nur aufgefüllt mit n/800-Methylamin.

III NaCl-Kontrolle.

\begin{tabular}{c|c|c|c|c}
\hline & v. & t. & p. & $\begin{array}{c}\text { Sauerstoff- } \\
\text { verbrauch } \\
\text { in ccm }\end{array}$ \\
\hline I 3 mal gewaschen & 37 & 17 & -24 & 0,084 \\
II aufgefüllt & 37 & 17 & -143 & 0,50 \\
III NaCl & 37 & 17 & -156 & 0,54
\end{tabular}

Dauer des Versuchs: 3 Stunden.

Resultat: $\mathrm{n} / \mathbf{8 0 0}$-Methylamin hemmt bei 3 maligem Waschen die Atmung um $84 \%$.

9. Nicotin.

Herstellung der n/so-n-Lösung: 
Über die Wirkung von Ammoniak und Ammoniakderivaten usw. 431

$$
\begin{array}{rl}
40,0 \mathrm{ccm} \text { einer } \mathrm{n} / 10-\text { Lösung Nicotin, } \\
80,0 \text { : } \mathrm{n} / \mathbf{1 0}-\mathrm{HCl}, \\
6,8 & 20 \% \mathrm{NaCl}, \\
86,0 & >\mathrm{NaOH} .
\end{array}
$$

Anordnung:

I 3 mal gewaschen.

II nur aufgefüllt mit $\mathrm{n} / 50$-Nicotin.

III NaCl-Kontrolle.

\begin{tabular}{c|c|c|c|c}
\hline \hline & v. & t. & p. & $\begin{array}{c}\text { Sauerstoff- } \\
\text { verbrauch } \\
\text { in ccm }\end{array}$ \\
\hline I 3 mal gewaschen & 37 & 16 & -47 & 0,16 \\
II aufgefüllt & 37 & 16 & -113 & 0,40 \\
III NaCl & 37 & 16 & -127 & 0,44
\end{tabular}

Dauer des Versuchs: 1 Std. 30 Min. Waschen.

Resultat: $\mathrm{n} / \mathrm{so}$-Nicotin hemmt die Atmung um $62 \%$ bei 3 maligem

$$
\text { 10. Amylamin. }
$$

Lösung: n/soo-Amylamin.

Anordnung:

I 3 mal gewaschen.

Il $1 \mathrm{mal}$ gewaschen und frisch aufgefullt.

III nur aufgefüllt mit n/200-Amylamin.

IV NaCl-Kontrolle.

\begin{tabular}{c|c|c|c|c}
\hline & v. & t. & p. & $\begin{array}{c}\text { Sauerstoff- } \\
\text { verbrauch } \\
\text { in ccm }\end{array}$ \\
\hline $\begin{array}{c}\text { I } 3 \text { mal gewaschen } \\
\text { II 1 mal gewaschen und } \\
\text { frisch gefüllt } \\
\text { III aufgefüllt }\end{array}$ & 37 & 15 & -27 & 0,095 \\
IV NaCl & 37 & 15 & -131 & 0,46 \\
& 37 & 15 & -155 & 0,54 \\
\end{tabular}

Dauer des Versuchs: 1 Std. 45 Min.

Resultat: n/800-Amylamin hemmt die Atmung um $77 \%$ bei dreimaligem Waschen.

11. Dimethylamin.

Lösung: $\mathbf{n} / \mathbf{s 0 0}$.

Anordnung:

I 3 mal gewaschen. 
II $1 \mathrm{mal}$ gewaschen und frisch aufgefüllt.

III nur aufgefüllt.

IV NaCl-Kontrolle.

\begin{tabular}{c|c|c|c|c}
\hline \hline & v. & t. & p. & $\begin{array}{c}\text { Sauerstoff- } \\
\text { verbrauch } \\
\text { in ccm }\end{array}$ \\
\hline $\begin{array}{c}\text { I 3 mal gewaschen } \\
\text { II 1 mal gewaschen und } \\
\text { frisch gefüllt }\end{array}$ & 37 & 15 & -15 & 0,053 \\
III aufgefüllt & 37 & 15 & -53 & 0,19 \\
IV NaCl & 37 & 15 & -120 & 0,42 \\
& & 15 & 0,31
\end{tabular}

Dauer des Versuchs: 1 Std. 20 Min.

Resultat: n/800-Dimethylamin hemmt die Atmung um $82 \%$ bei 3 maligem Waschen.

12. Allylamin.

Verwandt wird $n / 180-A l l y l a m i n$.

Anordnung: wie in den vorigen Versuchen.

\begin{tabular}{c|c|c|c|c}
\hline & v. & t. & p. & $\begin{array}{c}\text { Sauerstoff- } \\
\text { verbrauch } \\
\text { in ccm }\end{array}$ \\
\hline I 3 mal gewaschen & 37 & 16 & -36 & 0,12 \\
II 1 mal gewaschen und & 37 & 16 & -64 & 0,22 \\
frisch aufgefüllt & 37 & 16 & -149 & 0,52 \\
III aufgefüllt & 37 & 16 & -133 & 0,46 \\
IV NaCl &
\end{tabular}

Dauer des Versuchs: 1 Std. 30 Min.

Resultat: $\mathrm{n} / \mathbf{1 5 0}$-Allylamin hemmt die Atmung um $\mathbf{7 3} \%$ bei dreimaligem Waschen.

\section{Alkaloide.}

14. Brucin ( $M$ wasserfrei 394).

Versuch 14: Brucin.

Bereitung der Lösung : 0,6 g Brucin auf $300 \mathrm{ccm} \mathrm{0,9 \%} \mathrm{NaCl-Lösung,}$ heiß gelöst $=\mathrm{n} / 200$ Brucin.

Anordnung:

I 3 mal gewaschen.

II aufgefüllt.

III NaCl-Kontrolle. 


\begin{tabular}{c|c|c|c|c}
\hline & v. & t. & p. & $\begin{array}{c}\text { Sauerstoff- } \\
\text { verbrauch } \\
\text { in ccm }\end{array}$ \\
\hline I 3 mal gewaschen & 37 & 15 & -36 & 0,13 \\
II nur aufgefüllt & 37 & 15 & -95 & 0,33 \\
III NaCl & $\mathbf{3 7}$ & 15 & -100 & 0,35
\end{tabular}

Dauer des Versuchs 2 Std. 30 Min.

Resultat: n/200-Brucinlösung hemmt die Atmung um $64 \%$ bei 3 maligem Waschen.

$$
\text { 15. Chinin }(M=324) \text {. }
$$

Bereitung der Lösung: $0,7 \mathrm{~g}$ Chinin $+40 \mathrm{ccm} \mathrm{n/10-HCl,} \mathrm{davon}$ $6 \mathrm{cam}$ auf $300 \mathrm{ccm} \mathrm{0,9 \%} \mathrm{NaCl-Lösung} \mathrm{und} \mathrm{mit} \mathrm{der} \mathrm{äquivalenten} \mathrm{Menge}$

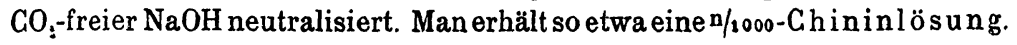

Anordnung:

I 3 mal gewaschen.

II nur aufgefüllt mit n/1000-Chinin.

III NaCl-Kontrolle.

\begin{tabular}{c|c|c|c|c}
\hline & v. & t. & p. & $\begin{array}{c}\text { Sauerstoff- } \\
\text { verbrauch } \\
\text { in ccm }\end{array}$ \\
\hline I 3 mal gewaschen & 37 & 15 & -26 & 0,091 \\
II aufgefüllt & 37 & 15 & -102 & 0,36 \\
III NaCl & 37 & 15 & -113 & 0,40
\end{tabular}

Dauer des Versuchs 2 Std. 30 Min..

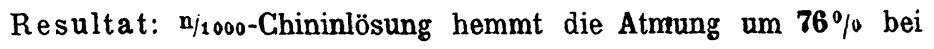
3 maligem Waschen.

$$
\text { 16. Codein }(M=317) \text {. }
$$

Bereitung der Lösung:

$$
\begin{aligned}
& \left.\begin{array}{c}
0,3 \mathrm{~g} \text { Codein } \\
40,0 \mathrm{ccm} \mathrm{n} / 10-\mathrm{HGl}
\end{array}\right\} \text { gut geschüttelt, } \\
& 3,4 \text {, } 20 \% \mathrm{NaCl} \text {, } \\
& 20,0>\mathrm{H}_{2} \mathrm{O} \text {, } \\
& 43,2>\mathrm{NaOH} \text {, } \\
& =\text { ca. } \mathrm{n} / 100-\text { Codein in } \mathrm{NaCl} \text {, } \\
& \text { verwandt wird n/400-Ciodein. }
\end{aligned}
$$

Anordnung:

I 4 mal gewaschen und frisch aufgefüllt.

II $2 \mathrm{mal}$ gewaschen und frisch aufgefüllt.

III nur aufgefüllt mit $\mathrm{n} / \mathbf{4 0 0}$-Codein.

IV NaCl-Kontrolle. 


\begin{tabular}{c|c|c|c|c}
\hline & v. & t. & p. & $\begin{array}{c}\text { Sauerstoff- } \\
\text { werbrauch } \\
\text { in ccm }\end{array}$ \\
\hline I 4 mal gewaschen und & 37 & 17 & -44 & 0,15 \\
$\begin{array}{c}\text { aufgefüllt } \\
\text { II 2 mal gewaschen und } \\
\text { frisch aufgefüllt } \\
\text { III aufgefüllt }\end{array}$ & 37 & 17 & -54 & 0,19 \\
IV NaCl & 37 & 17 & -72 & 0,25 \\
& 37 & 17 & -69 & 0,24
\end{tabular}

Dauer des Versuchs 2 Std. 40 Min.

Resultat: $\mathrm{n} / 400$-Codein hemmt die Atmung um $\mathbf{3 6} \%$ bei 4 maligem Waschen und Auffüllen mit frischer Lösung.

Verwandt $\mathrm{n} / 400-$ Cocain.

17. Gocain $(\mathrm{M}=303)$.

Anordnung:

I 3 mal gewaschen.

II $1 \mathrm{mal}$ gewaschen und frisch aufgefüllt.

III nur aufgefüllt.

IV NaCl-Kontrolle.

\begin{tabular}{|c|c|c|c|c|}
\hline & v. & t. & p. & $\begin{array}{l}\text { Sauerstoff- } \\
\text { verbrauch } \\
\text { in } \mathrm{ccm}\end{array}$ \\
\hline I $3 \mathrm{mal}$ gewaschen & 37 & 17 & -52 & 0,18 \\
\hline $\begin{array}{c}\text { II } 1 \text { mal gewaschen und } \\
\text { aufgefüllt }\end{array}$ & 37 & 17 & -92 & 0,32 \\
\hline III aufgefüllt & 37 & 17 & -118 & 0,41 \\
\hline IV $\mathrm{NaCl}$ & 37 & 17 & -92 & 0,32 \\
\hline
\end{tabular}

Dauer des Versuchs 2 Std. 30 Min. Waschen.

Resultat: $n / 400$-Cocain hemmt die Atmung um $43 \%$ bei 3 maligem

Lösung: n/400-Atropin.

Anordnung: Wie im vorigen Versuch.

\begin{tabular}{c|c|c|c|c}
\hline \hline & v. & t. & p. & $\begin{array}{c}\text { Sauerstoff- } \\
\text { verbrauch } \\
\text { in ccm }\end{array}$ \\
\hline I 3 mal gewaschen & 37 & 16 & -8 & 0,028 \\
II 1. mal gewaschen und & 37 & 16 & -74 & 0,26 \\
$\quad \begin{array}{c}\text { aufgefüllt } \\
\text { III aufgefüllt } \\
\text { IV NaCl }\end{array}$ & 37 & 16 & -103 & 0,36 \\
& 37 & 16 & -92 & 0,32
\end{tabular}

Dauer des Versuchs 2 Std. 30 Min. 
Über die Wirkung von Ammoniak und Ammoniakderivaten usw. 435

Resultat: $\mathrm{n} / 400-$ Atropin hemmt die Atmung um $90 \%$ bei 3 maligem Waschen.

\section{Untersuchangen zur Frage, ob die Atmungshemmung reversibel ist.}

\section{Ammoniak.}

Es soll untersucht werden, ob die durch Ammoniak hervorgerufene Atmungshemmung rückgängig gemacht werden kann.

Bereitung der Lösung wie in Versuch 5, jedoch mit «modifizierter Locke scher Lösung», da die Versuche über Salzlösungen die Schädigung durch $\mathrm{NaCl}$ gezeigt haben.

Anordnung: $\mathrm{n} / \mathrm{sb}-\mathrm{NH}_{3}$.

I 1 Röhrchen $2 \mathrm{ccm}$ Suspension + L o ck esche Lösung (mit $\mathrm{NaHCO}_{8}$ ).

II 2 Röhrchen $2 \mathrm{ccm}$ Suspension $+\mathrm{NH}_{3}$ in Lockescher Lösung (ohne $\mathrm{NaHCO}_{3}$ ).

III 1 Spitzglas $2 \mathrm{ccm}$ Suspension + Lock e sche Lösung (mit $\left.\mathrm{NaHCO}_{3}\right)$.

IV 2 Spitzglas $2 \mathrm{ccm}$ Suspension $+\mathrm{NH}_{3}$ gelöst in Locke (ohne $\mathrm{NaHCO}_{3}$ ).

Alle kommen für 2 Stunden in den Thermostaten von $29^{\circ}$, dann werden I und II in Eis gelegt.

Inhalt der Spitzgläser wird 2 mal mit Lockescher Lösung gewaschen, in Atemröhrchen gefüllt und für 2 Stunden in den Thermostaten gelegt.

\begin{tabular}{|c|c|c|c|c|}
\hline . & v. & t. & p. & $\begin{array}{l}\text { Sauerstoff- } \\
\text { verbrauch } \\
\text { in ccm }\end{array}$ \\
\hline $\begin{array}{c}\text { I Locke } 2 \text { Std. } \\
\text { II } \mathrm{NH}_{3} 2 \text { Std. }\end{array}$ & $\begin{array}{l}37 \\
37 \\
\end{array}$ & $\begin{array}{l}16 \\
16 \\
\end{array}$ & $\begin{array}{l}86 \\
25 \\
\end{array}$ & $\begin{array}{l}0,30 \\
0,087 \\
\end{array}$ \\
\hline $\begin{array}{l}\text { III Locke } 2 \cdot \text { e } 2 \text { Std. } \\
\text { IV mit Locke gewaschen } \\
2 \cdot \text { e } 2 \text { Std. }\end{array}$ & $\begin{array}{l}37 \\
37\end{array}$ & $\begin{array}{l}16 \\
16\end{array}$ & $\begin{array}{l}-85 \\
-80\end{array}$ & $\begin{array}{l}0,30 \\
0,28\end{array}$ \\
\hline
\end{tabular}

Resultat: Atmungshemmung in den ersten 2 Stunden von $\mathbf{7 0} \%$. Nach Waschen und den zweiten 2 Stunden Hemmung a uf $6 \%$ zurückgegangen.

\section{Ammoniak.}

Bereitung der Lösung, Anordnung und Dauer des Versuches genau wie vorher in 19 . 


\begin{tabular}{c|c|c|c|c}
\hline \hline & v. & t. & p. & $\begin{array}{c}\text { O-Verbrauch } \\
\text { in ccm }\end{array}$ \\
\hline I Locke 2 Std. & 37 & 17 & -87 & 0,30 \\
II NH 2 Std. & 37 & 17 & -28 & 0,097 \\
\hline III Locke 2. e 2 Std. & 37 & 17 & -87 & 0,30 \\
IV gewaschen 2. e 2 Std. & 37 & 17 & -79 & 0,27
\end{tabular}

Resultat: Die Atmung, die in den ersten 2 Stunden um $67 \%$ gehemmt war, steigt nach 3 maligem Waschen in den zweiten 2 Stunden fast wieder bis auf die ursprüngliche Höhe an.

\section{Godein.}

Bereitung der Lösung:

$0,4 \mathrm{~g}$ Codein auf $130 \mathrm{~g}$ Locke ohne $\mathrm{NaHCO}_{3}=\mathrm{ca}$. n/100-Codein.

Anordnung und Dauer des Versuches wie in Versuch 19 und 20.

\begin{tabular}{c|c|c|c|c}
\hline & v. & t. & p. & $\begin{array}{c}\text { O-Verbrauch } \\
\text { in ccm }\end{array}$ \\
\hline I Locke 2 Std. & 37 & 16 & -73 & 0,25 \\
II Codein 2 Std. & 37 & 16 & -10 & 0,035 \\
\hline III Locke 2 e 2 Std. & 37 & 16 & -70 & 0,24 \\
IV gewaschen 2 e 2 Std. & 37 & 16 & -77 & 0,27
\end{tabular}

Resultat: Die Atmung, die in den ersten 2 Stunden um $85 \%$ gehemmt war, ist nach den zweiten 2 Stunden völlig zur Norm zurückgekehrt.

E. Untersuchung einiger Stoffe von pharmakologischer Bedeutung. 22. Antipyrin und Coffein.

Anordnung:

I mit $1 \%$ Antipyrin $=$ ca. $\mathrm{n} / \mathbf{z o}$.

II mit $2 \%$ Antipyrin $=$ ca. $\mathrm{n} / \mathbf{1 0}$.

III mit $1 \%$ Coffein $=\mathrm{n} / \mathbf{2} 0$.

IV mit Lockescher Lösung.

Alle 1 mal gewaschen und mit frischer Lösung aufgefüllt.

Dauer des Versuchs: 3 Stunden.

\begin{tabular}{c|c|c|c|c}
\hline & v. & t. & p. & $\begin{array}{c}\text { O-Verbrauch } \\
\text { in ccm }\end{array}$ \\
\hline Antipyrin 1\% & 37 & 16 & -62 & 0,22 \\
Antipyrin 2\% & 37 & 16 & -45 & 0,16 \\
Coffein 1\% & 37 & 16 & -111 & 0,39 \\
Locke, Kontrolle & 37 & 16 & -109 & 0,38
\end{tabular}


Über die Wirkung von Ammoniak und Ammoniakderivaten usw.

Resultat: Unter den gegebenen Bedingungen hemmt 1\% Antipyrin um $42 \%, 2 \%$ um $58 \% ; 1 \%$ Coffein hemmt gar nicht.

23. Veronal und Sulfonal.

Anordnung:

I mit $0,5 \%$ Veronal $=$ ca. $\mathrm{n} / 30$.

II mit $0,2 \%$ Sulfonal $=$ ca. $\mathrm{n} / 110$.

III mit $\mathrm{NaCl}$.

Alle 1 mal gewaschen und frische Lösungen zugefüllt. Dann für 2 Std. 30 Min. in den Thermostaten.

\begin{tabular}{c|c|c|c|c}
\hline & v. & t. & p. & $\begin{array}{c}\text { O-Verbrauch } \\
\text { in ccm }\end{array}$ \\
\hline $0,5 \%$ Veronal & 37 & 16 & -34 & 0,12 \\
$0,2 \%$ Sulfonal & 37 & 16 & -93 & 0,32 \\
$\mathrm{NaCl}$ & 37 & 16 & -89 & 0,31
\end{tabular}

Resultat: Sulfonal hemmt in der Konzentration von $\mathbf{n} / 110$ nicht. Veronal hemmt um $61 \%$ unter den gegebenen Bedingungen.

\section{Veronal.}

Veronal in verschieden reagierenden Lösungen.

Anordnung:

I $100 \mathrm{ccm} 0,9 \% \mathrm{NaCl}+0,5 \mathrm{~g}$ Veronal $+24 \mathrm{n} / 10-\mathrm{NaOH}+0,06 \mathrm{~g}$ $\mathrm{NaCl}$ reagiert:

\section{gegen Neutralrot: gelb.}

\section{> Phenolphthalein: rosa.}

II $100 \mathrm{ccm} \mathrm{NaCl}(0,9 \%)+0,5 \mathrm{~g}$ Veronal $+24 \mathrm{ccm}$ einer $1,3 \%$ Bicarbonatlösung reagiert:

gegen Neutralrot: orange.

III $100 \mathrm{ccm} 0,9 \% \mathrm{NaCl}+0,5 \mathrm{~g}$ Veronal reagiert:

gegen Neutralrot: rot.

IV $0,9 \% \mathrm{NaCl}$.

, Phenolphthalein: farblos.

Lösung I, II, III, IV je zu $2 \mathrm{ccm}$ Suspension auf $20 \mathrm{ccm}$ zugefüllt, alle 2 mal gewaschen und für $2 \mathrm{Std}$. $30 \mathrm{Min}$. in den Thermostaten.

\begin{tabular}{ll|c|c|c|c}
\hline & & v. & t. & p. & $\begin{array}{c}\text { 0-Verbrauch } \\
\text { in ccm }\end{array}$ \\
\hline $0,5 \%$ Veronal in alkalischer Lösung & 37 & 15 & -102 & 0,36 \\
$0,5 \% 0$ > neutraler & $>$ & 37 & 15 & -70 & 0,25 \\
$0,5 \%$, saurer & , saCl & 37 & 15 & -36 & 0,13 \\
& & 37 & 15 & -82 & 0,29
\end{tabular}

Hoppe-Seyler's Zeitschrift f. physiol. Chemie. LXXIX. 
438 Eduard Grafe, Über die Wirkung von Ammoniak usw.

Resultat: $0,5 \%$ Veronal $=$ ca. $\mathrm{n} / \mathrm{\iota}$ hemmt in saurer Lösung um $\mathbf{5 5} \%$, in neutraler Lösung um 14\%, in alkalischer gar nicht.

25. Acetanilid und Phenacetin.

Anordnung:

I $0,5 \mathrm{~g}$ Acetanilid auf $100 \mathrm{ccm} 0,9 \% \mathrm{NaCl}$ heiß gelöst, ca. $\mathrm{n} / \mathbf{2 7}$. II $1 \mathrm{ccm}$ einer $10 \%$ igen alkoholischen Lösung Phenacetin auf $140 \mathrm{ccm} \mathrm{0,9 \%} \mathrm{NaCl-Lösung} \mathrm{ca.} \mathrm{n} / 250$.

III NaCl-Kontrolle.

Alle $3 \mathrm{mal}$ gewaschen und für 2 Std. $30 \mathrm{Min}$. in den Thermostaten.

\begin{tabular}{c|c|c|c|c}
\hline & v. & t. & p. & $\begin{array}{c}\text { O-Verbrauch } \\
\text { in ccm }\end{array}$ \\
\hline n/87-Acetanilid & 37 & 16 & 27 & 0,094 \\
n/850-Phenacetin & 37 & 16 & 53 & 0,18 \\
NaCl & 37 & 16 & 89 & 0,31
\end{tabular}
um $40 \%$. 